\title{
Crystal Structure of Dimethyl[4-tert-butyl-2,6-bis(1- naphthyliminomethylene)phenolato]gallium
}

\author{
Wei-Jin Gu,*** Ying-zhong Shen,*** Yi-Zhi LI,* Yi PAN*广 and Cheng-Jian ZHU* \\ * Coordination Chemistry Institute, State Key Laboratory of Coordination Chemistry, \\ Nanjing University, Nanjing 210093, P. R. China \\ **Department of Chemistry, Nanjing Normal University, Nanjing 210097, P. R. China \\ ***School of Material Science \& Technology, Nanjing University of Aeronautics \& \\ Astronautics, Nanjing, 210016, P. R. China
}

\begin{abstract}
Crystals of dimethyl [4-tert-butyl-2,6-bis(1-naphthyliminomethylene)phenolato]gallium (1) are monoclinic, space group $P 2{ }_{1} / c$ with $a=14.797(3) \AA, b=10.651(2) \AA, c=20.260(4) \AA, \beta=93.55(1)^{\circ}, V=3187.1(11) \AA^{3}, Z=4$. The structure was solved by direct methods and refined to a final $R$ value of 0.0412 of 4150 reflections $(I>2 \sigma(I))$. The gallium atom was bonded by one oxygen atom and coordinated by one imine nitrogen atom, forming one six-member ring.
\end{abstract}

(Received October 12, 2004; Accepted January 6, 2005; Published on web April 22, 2005)

The chemistry of organoaluminum, gallium and indium compounds have received renewed interests in recent years due to their wide applications in material science $^{1}$ and organic reactions. ${ }^{2}$ Among them, one of the major categories of the compounds is organometallic alkoxides or phenoxides. We recently synthesized the title compound $\mathbf{1}$ by using trimethylgallium reacting with 4-tert-butyl-2,6-bis(1naphthyliminomethylene)pheno ${ }^{3}$ at room temperature. The hydroxyl in phenol reacts with trimethylgallium by removing one molecule of methane as a by-product, as shown in Fig. 1.

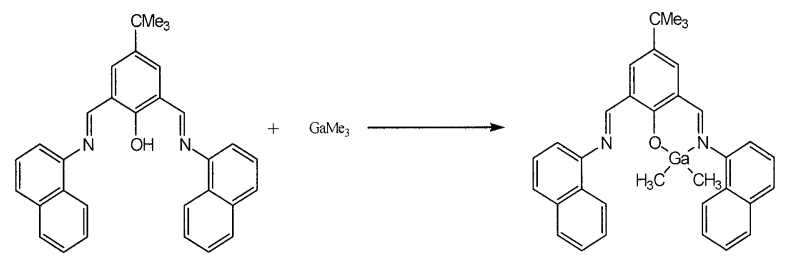

Fig. 1 Synthesis and chemical structure of the title compound $\mathbf{1}$.

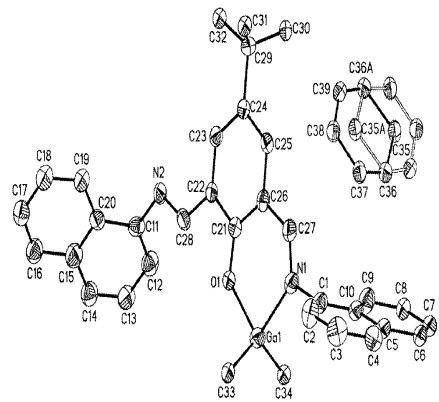

Fig. 2 Molecular structure of (1) showing $50 \%$ probability displacement ellipsoids along with the numbering scheme.

$†$ To whom correspondence should be addressed.

E-mail: yipan@netra.nju.edu.cn
Single crystals for X-ray analysis were obtained by the slow evaporation of a benzene solution at room temperature. All of the non-hydrogen atoms were refined anisotropically. The positions of the hydrogen atoms were determined from a difference Fourier map, and riding models were applied on refinements. The crystal and experimental data are given in Table 1. The final fractional atomic coordinates and the equivalent isotropic thermal parameter for the non-hydrogen atoms are given in Table 2. Selected bond distances and bond

Table 1 Crystal and experimental data

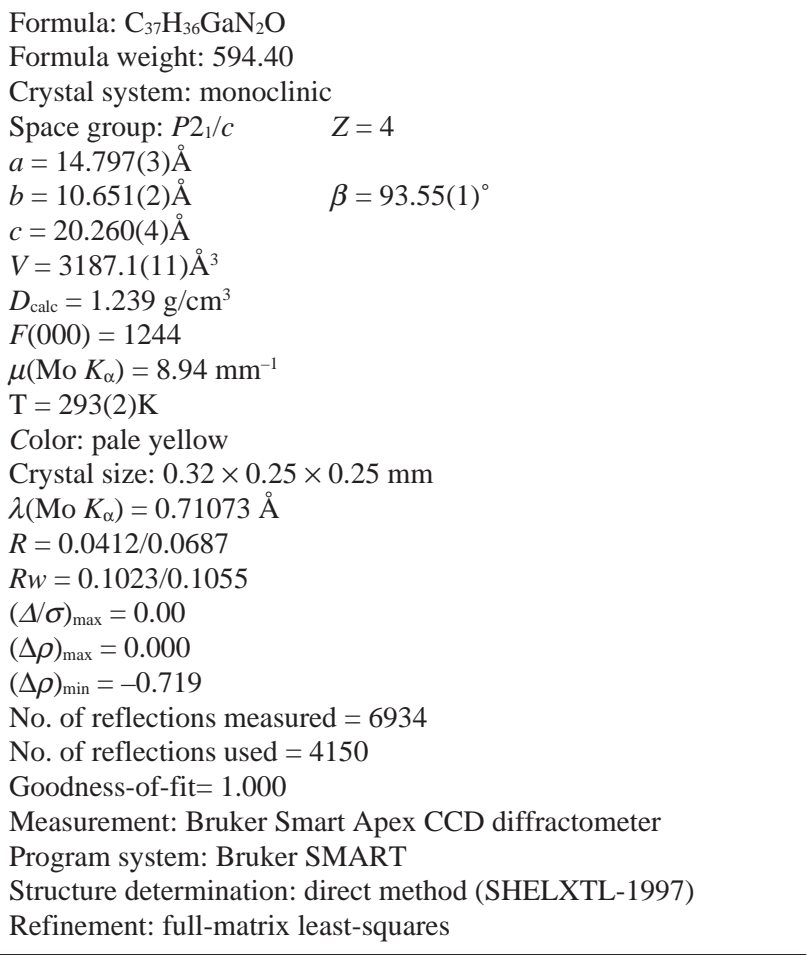


Table 2 Final atomic coordinates and equivalent isotropic thermal parameters $\left(U_{\mathrm{eq}}\right)$ for non-hydrogen atoms

\begin{tabular}{|c|c|c|c|c|}
\hline Atom & $x$ & $y$ & $z$ & $U_{\mathrm{eq}}\left(\AA^{2}\right)$ \\
\hline $\mathrm{Ga}(1)$ & $0.131450(19)$ & $0.11708(3)$ & $0.444530(14)$ & $0.05314(12)$ \\
\hline $\mathrm{N}(1)$ & $0.24255(14)$ & $0.22996(19)$ & $0.44244(10)$ & $0.0533(5)$ \\
\hline $\mathrm{N}(2)$ & $0.23245(13)$ & $-0.23016(19)$ & $0.66121(10)$ & $0.0497(5)$ \\
\hline$C(1)$ & $0.24492(17)$ & $0.3297(2)$ & $0.39256(13)$ & $0.0526(7)$ \\
\hline C(2) & $0.26969(18)$ & $0.2997(3)$ & $0.33170(13)$ & $0.0593(7)$ \\
\hline$C(3)$ & $0.27656(19)$ & $0.3928(3)$ & $0.28399(14)$ & $0.0621(7)$ \\
\hline $\mathrm{C}(4)$ & $0.25441(18)$ & $0.5158(3)$ & $0.29841(13)$ & $0.0576(7)$ \\
\hline $\mathrm{C}(5)$ & $0.22624(17)$ & $0.5462(2)$ & $0.36117(13)$ & $0.0517(6)$ \\
\hline C(6) & $0.20336(18)$ & $0.6689(3)$ & $0.37756(15)$ & $0.0618(7)$ \\
\hline$C(7)$ & $0.1762(2)$ & $0.6972(3)$ & $0.43718(15)$ & $0.0678(8)$ \\
\hline C ( 8$)$ & $0.1712(2)$ & $0.6084(3)$ & $0.48560(14)$ & $0.0666(8)$ \\
\hline $\mathrm{C}(9)$ & $0.19111(18)$ & $0.4838(3)$ & $0.47308(13)$ & $0.0585(7)$ \\
\hline $\mathrm{C}(10)$ & $0.22171(17)$ & $0.4504(3)$ & $0.40882(13)$ & $0.0555(7)$ \\
\hline C(11) & $0.15741(17)$ & $-0.3095(2)$ & $0.67336(12)$ & $0.0512(6)$ \\
\hline $\mathrm{C}(12)$ & $0.07572(18)$ & $-0.2618(3)$ & $0.68849(13)$ & $0.0579(7)$ \\
\hline$C(13)$ & $0.00269(19)$ & $-0.3417(3)$ & $0.69638(14)$ & $0.0642(8)$ \\
\hline$C(14)$ & $0.01046(19)$ & $-0.4652(3)$ & $0.68947(13)$ & $0.0629(8)$ \\
\hline $\mathrm{C}(15)$ & $0.09296(19)$ & $-0.5186(2)$ & $0.67501(12)$ & $0.0562(7)$ \\
\hline$C(16)$ & $0.1054(2)$ & $-0.6511(3)$ & $0.66550(14)$ & $0.0656(8)$ \\
\hline$C(17)$ & $0.1857(2)$ & $-0.6995(3)$ & $0.65272(14)$ & $0.0690(8)$ \\
\hline$C(18)$ & $0.2593(2)$ & $-0.6235(3)$ & $0.64905(14)$ & $0.0666(8)$ \\
\hline$C(19)$ & $0.25228(19)$ & $-0.4969(3)$ & $0.65594(13)$ & $0.0567(7)$ \\
\hline $\mathrm{C}(20)$ & $0.16947(18)$ & $-0.4422(2)$ & $0.66878(12)$ & $0.0499(6)$ \\
\hline $\mathrm{C}(21)$ & $0.26267(18)$ & $0.0301(2)$ & $0.54630(13)$ & $0.0497(6)$ \\
\hline $\mathrm{C}(22)$ & $0.28651(17)$ & $-0.0525(2)$ & $0.60059(12)$ & $0.0475(6)$ \\
\hline C(23) & $0.36855(17)$ & $-0.0423(2)$ & $0.63555(12)$ & $0.0479(6)$ \\
\hline C (24) & $0.43284(17)$ & $0.0484(2)$ & $0.62335(12)$ & $0.0482(6)$ \\
\hline C(25) & $0.40858(17)$ & $0.1322(2)$ & $0.57263(12)$ & $0.0492(6)$ \\
\hline$C(26)$ & $0.32643(17)$ & $0.1252(2)$ & $0.53438(12)$ & $0.0494(6)$ \\
\hline$C(27)$ & $0.31210(17)$ & $0.2173(2)$ & $0.48300(13)$ & $0.0527(7)$ \\
\hline C(28) & $0.22002(17)$ & $-0.1470(2)$ & $0.61730(12)$ & $0.0497(6)$ \\
\hline C(29) & $0.52457(17)$ & $0.0600(2)$ & $0.66279(13)$ & $0.0501(6)$ \\
\hline$C(30)$ & $0.53386(17)$ & $0.1846(2)$ & $0.69538(12)$ & $0.0523(6)$ \\
\hline $\mathrm{C}(31)$ & $0.60084(17)$ & $0.0551(2)$ & $0.61527(12)$ & $0.0533(7)$ \\
\hline C(32) & $0.53708(17)$ & $-0.0429(2)$ & $0.71281(12)$ & $0.0526(7)$ \\
\hline$C(33)$ & $0.12449(17)$ & $0.0214(2)$ & $0.36278(12)$ & $0.0514(6)$ \\
\hline C(34) & $0.03354(16)$ & $0.2079(2)$ & $0.48224(12)$ & $0.0518(6)$ \\
\hline$O(1)$ & $0.18765(11)$ & $0.01467(14)$ & $0.51193(8)$ & $0.0506(4)$ \\
\hline$C(36)$ & $0.45023(17)$ & $0.5385(2)$ & $0.45670(13)$ & $0.0498(6)$ \\
\hline C(38) & $0.5496(3)$ & $0.3684(5)$ & $0.4345(3)$ & $0.0521(13)$ \\
\hline$C(37)$ & $0.4824(3)$ & $0.4576(5)$ & $0.4150(3)$ & $0.0521(13)$ \\
\hline C(39) & $0.5810(4)$ & $0.3812(5)$ & $0.5006(3)$ & $0.0525(13)$ \\
\hline$C(35)$ & $0.4837(4)$ & $0.5474(5)$ & $0.5224(3)$ & $0.0526(13)$ \\
\hline
\end{tabular}

$U_{\mathrm{eq}}=1 / 3 \Sigma_{i} \Sigma_{j} U_{i} a_{i}^{*} a_{j}^{*}\left(a_{i} a_{j}\right)$

angles are listed in Table 3.

The results clearly show that the gallium atom is fourcoordinate. The coordination sphere of the central gallium atoms can be described as a distorted tetrahedron. A benzene molecule is found on an inversion center, and is disordered into two sites with an occupancy of 0.5 each. The Ga1-O1 distance [1.8988(16) $\mathrm{A}]$ is shorter than that of dimethyl[ $N$-salicylidene 2aminopyridine]gallium [1.917(2) $\mathrm{A}](\mathbf{2}){ }^{4}$ The Ga1-N1 distances is $2.039(2) \AA$, which is shorter than that of 2 [2.059(2) $\AA$ ] ${ }^{4}$ One
Table 3 Selected bond distances $(\AA)$, angles $\left({ }^{\circ}\right)$

\begin{tabular}{llll}
\hline Bond distance & & & \\
$\mathrm{Ga}(1)-\mathrm{O}(1)$ & $1.8988(16)$ & $\mathrm{Ga}(1)-\mathrm{C}(34)$ & $1.938(2)$ \\
$\mathrm{Ga}(1)-\mathrm{C}(33)$ & $1.942(2)$ & $\mathrm{Ga}(1)-\mathrm{N}(1)$ & $2.039(2)$ \\
$\mathrm{N}(1)-\mathrm{C}(27)$ & $1.284(3)$ & $\mathrm{N}(1)-\mathrm{C}(1)$ & $1.468(3)$ \\
$\mathrm{N}(2)-\mathrm{C}(28)$ & $1.261(3)$ & $\mathrm{C}(21)-\mathrm{O}(1)$ & $1.284(3)$ \\
& & & \\
Bond angles & & & \\
& & & \\
$\mathrm{O}(1)-\mathrm{Ga}(1)-\mathrm{C}(34)$ & $107.95(9)$ & $\mathrm{O}(1)-\mathrm{Ga}(1)-\mathrm{C}(33)$ & $108.03(9)$ \\
$\mathrm{C}(34)-\mathrm{Ga}(1)-\mathrm{C}(33)$ & $126.65(10)$ & $\mathrm{O}(1)-\mathrm{Ga}(1)-\mathrm{N}(1)$ & $92.06(8)$ \\
$\mathrm{C}(34)-\mathrm{Ga}(1)-\mathrm{N}(1)$ & $109.70(9)$ & $\mathrm{C}(33)-\mathrm{Ga}(1)-\mathrm{N}(1)$ & $106.98(10)$ \\
$\mathrm{C}(27)-\mathrm{N}(1)-\mathrm{C}(1)$ & $117.7(2)$ & $\mathrm{C}(27)-\mathrm{N}(1)-\mathrm{Ga}(1)$ & $122.69(17)$ \\
$\mathrm{C}(1)-\mathrm{N}(1)-\mathrm{Ga}(1)$ & $119.65(16)$ & $\mathrm{C}(28)-\mathrm{N}(2)-\mathrm{C}(11)$ & $117.1(2)$ \\
$\mathrm{C}(10)-\mathrm{C}(1)-\mathrm{N}(1)$ & $119.6(2)$ & $\mathrm{C}(12)-\mathrm{C}(11)-\mathrm{N}(2$ & $121.9(2)$ \\
$\mathrm{N}(2)-\mathrm{C}(11)-\mathrm{C}(20)$ & $118.2(2)$ & $\mathrm{O}(1)-\mathrm{C}(21)-\mathrm{C}(26)$ & $124.2(2)$ \\
$\mathrm{O}(1)-\mathrm{C}(21)-\mathrm{C}(22)$ & $120.0(2)$ & $\mathrm{C}(21)-\mathrm{C}(22)-\mathrm{C}(28)$ & $117.6(2)$ \\
$\mathrm{N}(1)-\mathrm{C}(27)-\mathrm{C}(26)$ & $127.7(2)$ & $\mathrm{C}(21)-\mathrm{C}(26)-\mathrm{C}(27)$ & $122.8(2)$ \\
$\mathrm{N}(2)-\mathrm{C}(28)-\mathrm{C}(22)$ & $125.2(2)$ & $\mathrm{C}(21)-\mathrm{O}(1)-\mathrm{Ga}(1)$ & $129.93(15)$ \\
& & & \\
& & &
\end{tabular}

Estimated standard deviations in the least-significant figure are given in parentheses.

nitrogen atom coordinates to the gallium atom, forming one irregular six-member ring (Ga1-N1-C27-C26-C21-O1). In the ring, the $\mathrm{O} 1-\mathrm{Ga} 1-\mathrm{N} 1$ angle is $92.06(8)^{\circ}$, which is larger than that of $2\left(90.76(7)^{\circ}\right) .^{4}$

\section{Acknowledgements}

We gratefully acknowledge the National Natural Science Foundation of China. The research grants for Y. Pan from the Qin-Lian Program of Jiangsu Province, the Kua-shi-ji Program of the Education Ministry and the National 863 High Technology Program is also acknowledged.

\section{References}

1. Y. Z. Shen, H. W. Gu, W. J. Gu, F. Yuan, Y. Zhu, and Y. Pan, J. Organometal. Chem., 2003, 681, 51.

2. M. S. Taylor and E. N. Jacobsen, J. Am. Chem. Soc., 2003, 125,11204

3. L. Y. Wang, W. H. Sun, L. Q. Han, Z. L. Li, Y. L. Hu, C. C. He, and C. H. Yan, J. Organomet. Chem., 2002, 650, 59.

4. Y. Z. Shen, H. W. Gu, Y. Pan, G. Dong, X. Y. Huang, and H. W. Hu, J. Organomel. Chem., 2000, 605, 234. 